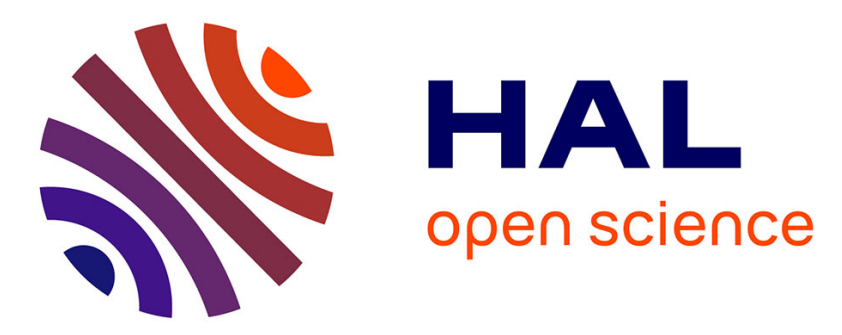

\title{
kHz-speed optically induced phase gratings with liquid crystal light valves in transient dynamic mode
}

Umberto Bortolozzo, Stefania Residori, Jean-Pierre Huignard

\section{To cite this version:}

Umberto Bortolozzo, Stefania Residori, Jean-Pierre Huignard. kHz-speed optically induced phase gratings with liquid crystal light valves in transient dynamic mode. Optics Letters, 2021, 46 (19), pp.4730-4733. 10.1364/OL.438289 . hal-03431891

\section{HAL Id: hal-03431891 \\ https://hal.science/hal-03431891}

Submitted on 17 Nov 2021

HAL is a multi-disciplinary open access archive for the deposit and dissemination of scientific research documents, whether they are published or not. The documents may come from teaching and research institutions in France or abroad, or from public or private research centers.
L'archive ouverte pluridisciplinaire HAL, est destinée au dépôt et à la diffusion de documents scientifiques de niveau recherche, publiés ou non, émanant des établissements d'enseignement et de recherche français ou étrangers, des laboratoires publics ou privés. 


\title{
kHz-speed optically induced phase gratings with liquid crystal light valves in transient dynamic mode
}

\author{
Umberto Bortolozzo, ${ }^{1}$ Stefania Residor ${ }^{1}{ }^{1,}$, JeAN-PIERRe HuIgNard ${ }^{2}$ \\ ${ }^{1}$ HOASYS SAS, 1047 route des Dolines, Business Pôle, 06560 Valbonne, France \\ 2 Jphopto-consultant, 20 rue Campo Formio, 75013 Paris, France \\ *Corresponding author: stefania.residori@hoasys.fr
}

Received XX Month XXXX; revised XX Month, XXXX; accepted XX Month XXXX; posted XX Month XXXX (Doc. ID XXXXX); published XX Month XXXX

Liquid crystal light valves (LCLV) are optically addressable spatial light modulators that allow controlling the phase and amplitude properties of optical beams. We show that submilliseconds phase and amplitude modulations can be obtained when operating the LCLV in the transient dynamic mode by setting the working point close to the saturation of the response. Thanks to the large birefringence of the liquid crystals, this condition provides enough phase shifts to respond to the needs of several methods for, optical measurement, dynamic holography, interferometry and imaging through phase disturbing media, while providing $\mathrm{kHz}$ speed. These values of response times also allow foreseeing applications, for example in biophotonics, and for monitoring of the environment.

Liquid crystal (LC) devices have large applications, for example in image displays and light projection [1], and also the LC Spatial light modulators SLM are now extensively used for 2D laser wavefront beam shaping [2]. According to the response time of the LC the typical bandwidth is $\sim 100 \mathrm{~Hz}$, corresponding to the large refractive index changes of the order of $\sim 0.1$. In LC cell with planar anchoring the index of refraction depends on the applied bias voltage $V_{b}$. A full birefringence excursion occurs from a few Volts (Fréedericksz transition [3] voltage $V_{F}$ ) to $\sim 10 \mathrm{~V}$ when all the molecules tend to align perpendicular to the cell wall. Once the field is removed, the fall time, which is the intrinsic relaxation time of the molecular orientation, is given by

$$
T=\frac{\gamma_{1} d^{2}}{K \pi^{2}},
$$

where $\gamma_{1}$ is the LC rotational viscosity, $K$ the elastic constant and $d$ the thickness of the LC layer. When applying the field, the rise time is related to the applied bias voltage through the equation [1]

$$
T_{O N}=\frac{\gamma_{1} d^{2}}{K \pi^{2}\left[\left(\frac{V_{b}}{V_{F}}\right)^{2}-1\right]},
$$

Hence, for $V_{b}>>V_{F}$ it can be taken as $T_{O N} \sim T\left(V_{F} / V_{b}\right)^{2}$ [4]. A full $\mathrm{ON} / \mathrm{OFF}$ excursion of the molecular reorientation requires a total switching time $T_{\text {tot }} \sim T\left[\left(V_{F} / V_{b}\right)^{2}+1\right]$. To decrease the fall time $T$, one can act on the LC parameters, for instance approaching the isotropic phase by increasing the temperature [5], or can diminish the LC thickness. However, the rise time Ton can be more simply decreased by increasing the applied voltage $V_{b}>>V_{F}$. Among the various approaches for decreasing the response time of a single LC modulator, the transient dynamics effect has been proposed and analyzed by ST Wu et al [6]. It is based on the idea of keeping the cell constantly biased at $\mathrm{ON}$ with the voltage $V_{b}$ at an intermediated value of the cell full transmittance response, and by removing the low modulation voltage $\Delta \mathrm{V}<V_{b}$ during the OFF part of the cycle [6]. In this transient dynamic mode the modulator exploits the small angle relaxation of a highly-deformed LC director $[7,8]$.

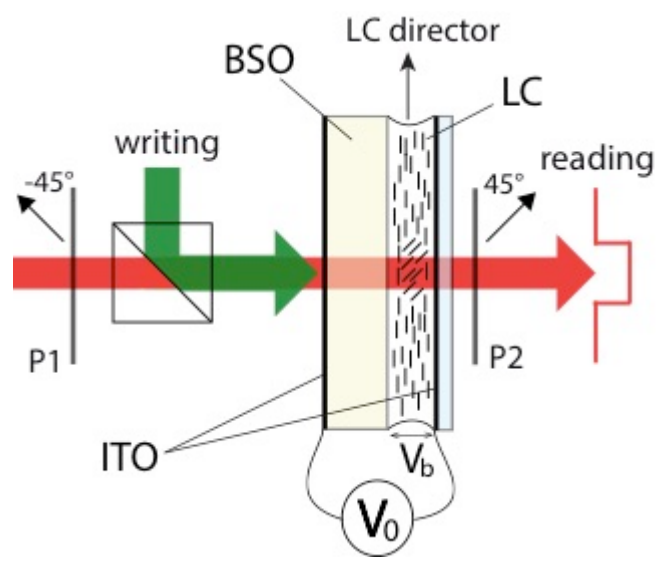

Fig. 1. Schematic structure of the LCLV. ITO : transparent conductive layer. BSO : photoconductive substrate. LC : planar aligned nematic liquid crystal layer, thickness $25 \mu \mathrm{m}$. $V_{0}$ : externally applied voltage. $\mathrm{Vb}$ : LC transferred bias voltage due to $\mathrm{V}_{0}$ and LCLV green light illumination. P1, P2 : crossed polarizers at $\pm 45^{\circ}$ with the LC director. 
Here, we show that by constantly applying a high bias voltage to a liquid crystal light valve (LCLV), and operating the cell in the transient mode effect, a fast $(\sim \mathrm{ms})$ switching time can be obtained, despite the relatively thick $25 \mu \mathrm{m}$ LC layer. In the LCLV the bias voltage $V_{b}$ is due to the low intensity laser illumination of a photoconductive crystal. In this component the transient mode effect exploits the saturation part of the optical response. Thanks to the reduced molecular excursion of the LC sub-milliseconds response times are achieved when dealing with small amplitude photo-induced phase modulations.

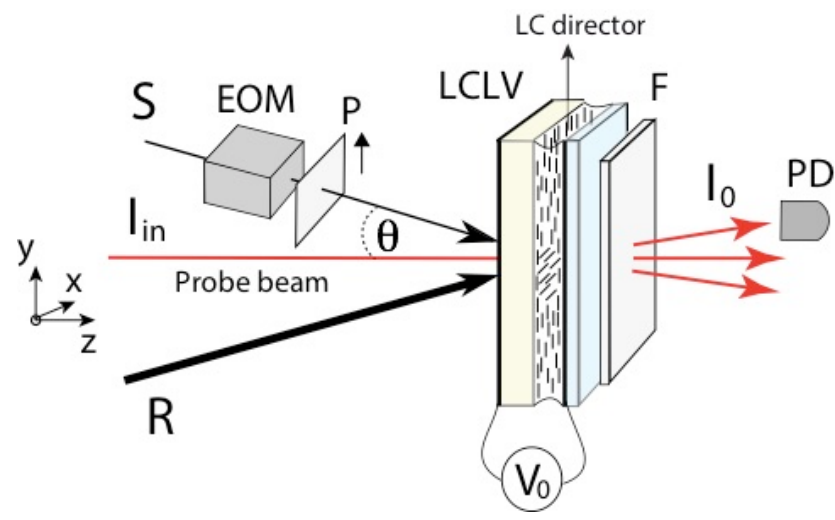

Fig. 2. Two-wave mixing in the LCLV: reference $R$ and signal $S$ at wavelength $532 \mathrm{~nm}$ interfere in the LC layer giving rise to a refractive index grating. The red probe beam $I_{\text {in }}$ reads the phase grating. $I_{0}$ : first diffracted order. EOM : electro-optic modulator. P : polarizer. F : blocking filter for green. PD : photodiode. Beam polarizations are along y axis, parallel to the LC director.

The schematic structure of the LCLV is represented in Fig.1. Liquid crystal light valves (LCLV) are optically addressable spatial light modulators, in which a continuous photosensitive layer is used to drive the orientation of a nematic LC layer [2]. Applications include dynamic holography [9], slow light interferometry [10,11] and optical sensing [12]. By using a LCLV fabricated with a semiconductor $\mathrm{Bi}_{12} \mathrm{SiO}_{20}$ crystal (BSO) as a photoconductor, we show that the voltage working point can be set close to the saturation of the optical response, in the conditions where $V_{b} \gg>V_{F}$ providing a reduced molecular excursion and hence, a faster response time. Here the LCLV operates with small refractive index modulation due to low holographic fringe contrast.

In the LCLV the photoconductive BSO layer is associated with a planar aligned nematic liquid crystal layer of E48 (from Merck). The extraordinary and ordinary index are, respectively, $n_{\mathrm{e}} \sim 1.75$ and $n_{\mathrm{o}} \sim 1.52$ at $589 \mathrm{~nm}$, for a total birefringence $n_{\mathrm{e}}-n_{\mathrm{o}} \sim 0.23$. The thickness of the LC layer is $d=25 \mu \mathrm{m}$. Transparent conductive electrodes of Indium-Tin-Oxide (ITO) are deposited on the outer side of the BSO and inner side of the glass wall closing the cell. An external voltage $V_{0}$ is applied across the LCLV and sets the bias voltage $V_{b}$ across the LC layer. Note that $V_{b}<<V_{0}$ without LCLV illumination due to the high dark resistivity of the BSO crystal, therefore, in the dark the most of the applied voltage is on the BSO [13]. On the other hand, when the BSO is illuminated its resistivity decreases due to the photoconductive effect and, correspondingly, $\mathrm{V}_{\mathrm{b}}$ increases across the LC layer.

The BSO is sensitive in the blue-green region of the visible spectrum. Therefore, a writing light beam in this wavelength range induces a molecular LC reorientation when impinging on the BSO, thanks to the locally reduced resistivity of the BSO and consequent increase of the local bias voltage. Correspondingly, a reading laser beam acquires a phase shift when passing through the LC layer. The phase shift acquired by the reading beam is optically controlled by the writing green beam intensity. Note that phase changes can be converted into amplitude variations by inserting the LCLV in between crossed polarizers at $\pm 45^{\circ}$ with respect to the LC director. Based on the writing-reading transcription process, phase and/or amplitude modulations can be transferred from the writing beam to the reading beam. For example, phase/amplitude modulations can be transformed at another laser wavelength or at another optical intensity. The dynamics of the process is driven by the LC response time, as expressed in Eq.(1).

The usual way of transferring amplitude/phase information is to perform two-wave mixing. In Fig.2 it is shown the setup to perform two-wave mixing in the LCLV, the main scheme used for holographic interferometry and vibrometry, involving a large reference to signal beam ratio and consequently a low fringe index modulation regime. A plane wave signal $S$ and a reference beams $R$ interfere onto the photosensitive BSO layer of the LCLV. In the experiment here presented the wavelength of $\mathrm{R}$ and $\mathrm{S}$ is $\lambda=532 \mathrm{~nm}$ and the grating spacing $\Lambda=\lambda /(2 \sin \theta) \sim 30 \mu \mathrm{m}$. The beam intensity ratio is $\beta=\mathrm{I}_{R} / \mathrm{I}_{\mathrm{S}} \sim 20$, with $\mathrm{I}_{\mathrm{S}}<\mathrm{I}_{\mathrm{R}}$ and the reference intensity $\mathrm{I}_{\mathrm{R}}=8 \mathrm{~mW} / \mathrm{cm}^{2}$.

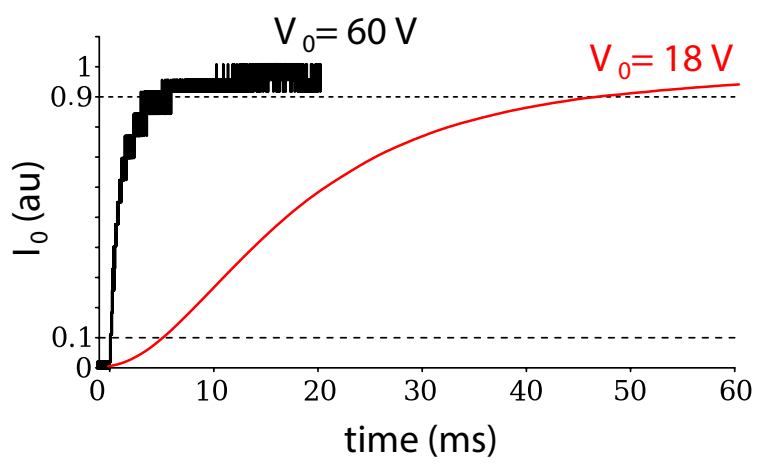

Fig. 3. Normalized step responses of the LCLV measured for $\mathrm{V}_{0}=18 \mathrm{Vrms}$ (red longer curve) and $\mathrm{V}_{0}=60 \mathrm{Vrms}$ (black shorted curve) by switching $\mathrm{ON}$ the signal beam. The intensity of the reference beam is $I_{R}=8 \mathrm{~mW} / \mathrm{cm}^{2}$. The curves are normalized to 1 for comparison.

The fringe intensity on the photoconductor is given by

$$
I_{L C L V}=I_{R}+2|S||R| \cos \left(\frac{2 \pi}{\Lambda} x+\Delta \varphi\right),
$$

where the signal intensity has been neglected, $\mathrm{I}_{\mathrm{S}}<<\mathrm{I}_{\mathrm{R}}, \Delta \varphi$ is the phase shift between $S$ and $R$ and the spatial coordinate $\mathrm{x}$ is along the LC director axis. Correspondingly, this intensity distribution produces a small angle $L C$ reorientation and a refractive index

$$
n=n_{2}\left(I_{R}+2|S||R| \cos \left(\frac{2 \pi}{\Lambda} x+\Delta \varphi\right)\right),
$$

where $\mathrm{n}_{2}$ is the Kerr-like coefficient of the LCLV [12]. The light intensity induces a refractive index grating proportional to $2 n_{2}|S||R|$. We call $\Delta n$ the amplitude modulation of this phase grating. Note that the value of $\mathrm{n}_{2}$ can be evaluated by directly 
measuring the light induced phase shift in an interferometer setup or by measuring the two-wave mixing gain. Typical values of $\mathrm{n}_{2}$ for the LCLV in the green are of the order of $6 \mathrm{~cm}^{2} / \mathrm{W}$ [12].

The grating amplitude $\Delta n$ depends on the modulation frequency of the signal beam. In general, the signal and reference beams are themselves self-diffracted by the induced grating and, due to their mutual coupling, exchange phase and amplitude modulation information. This allows transforming the signal phase modulations into intensity variations at the output diffracted beams by the LCLV (see, e.g., [12] and references therein).

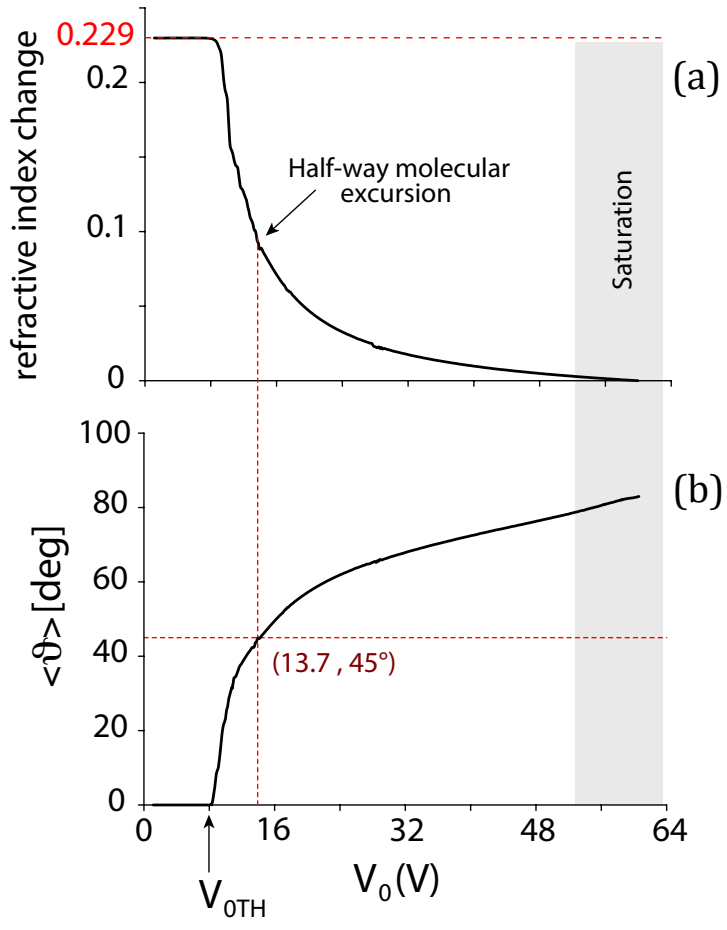

Fig. 4. (a) Refractive index change and (b) corresponding LC molecule average orientation angle $<\theta>$ vs amplitude of the applied voltage $V_{0}$ (in Vrms).

As a first experiment a step response is measured by switching on/off the signal $S$ with a constant bias voltage $V_{b}$. The amplitude of the refractive index grating is measured by using a probe beam $\mathrm{I}_{\text {in }}$ issued from a laser diode, wavelength $\lambda_{0}=670 \mathrm{~nm}$. This is done at two different bias voltages, the first one in the usual reorientational regime, $V_{0}=18 \mathrm{Vrms}$, the other one in the saturation regime, $\mathrm{V}_{0}=60 \mathrm{Vrms}$. These values allows to report across the LC layer a bias voltage $\mathrm{V}_{\mathrm{b}} \sim 12$ Vrms and $\sim 54 \mathrm{Vrms}$, which are, respectively, 8 and 36 times the threshold voltage $V_{F} \sim 1.5 \mathrm{Vrms}$. In both cases the reference to signal ratio is fixed to $\beta=20$. In Fig.3 are shown the step responses obtained by switching ON the signal. For comparison, the diffracted probe intensities $\mathrm{I}_{0}$ are normalized in between 0 and 1 and are plotted versus time for the two bias voltages, respectively. The corresponding rise time is $T_{\text {on }}=43 \mathrm{~ms}$ $\left(\mathrm{V}_{0}=18 \mathrm{~V}\right)$ and $T_{o n}=2.9 \mathrm{~ms} \quad\left(\mathrm{~V}_{0}=60 \mathrm{~V}\right)$ and the maximum index change reached at the steady state is $7 \times 10^{-3}$ and $10^{-3}$, respectively. The corresponding probe diffraction efficiencies on $\mathrm{I}_{0}$ are $\eta=0.33$ $\left(\mathrm{V}_{0}=18 \mathrm{~V}\right)$ and $\eta=1,3 \times 10^{-2}\left(\mathrm{~V}_{0}=60 \mathrm{~V}\right)$, respectively. The diffraction efficiency is related to the grating amplitude through the expression

$$
\eta \equiv \frac{I_{0}}{I_{\text {in }}}=\left|J_{1}\left(\frac{2 \pi d}{\lambda_{0}} \Delta n\right)\right|^{2},
$$

where $J_{1}$ is the first order Bessel function of the first kind.

When taking into account the fall time $T$, the total switching time can be estimated as $T_{\text {tot }} \sim 8 \mathrm{~ms}$ for $\mathrm{V}_{0}=60 \mathrm{~V}$ and $T_{\text {tot }} \sim 90 \mathrm{~ms}$ for $\mathrm{V}_{0}=18 \mathrm{~V}$. The gain in the response speed is counterbalanced by a smaller refractive index modulation, because the LCLV operates around different working points on the characteristics of the LC response. However, the maximum index change, as shown in the step response, provides sufficient dynamics for detecting the phase modulation and the efficiency of the induced holographic grating.

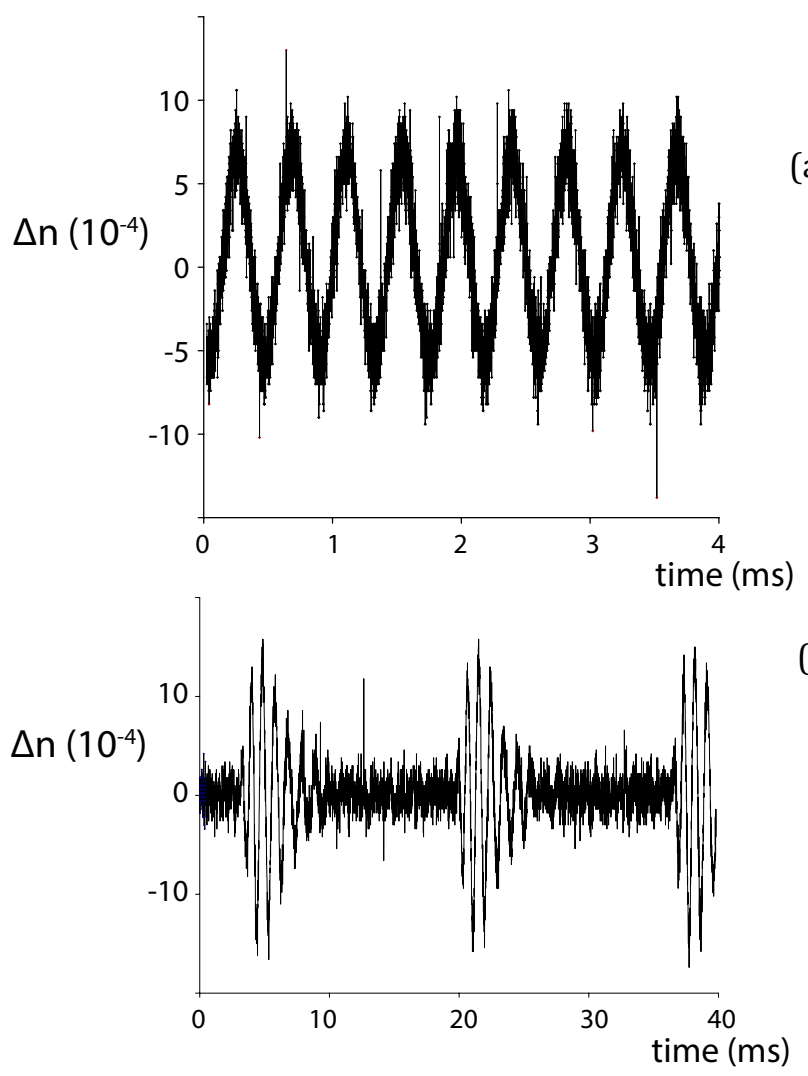

Fig. 5. Sub-millisecond amplitude modulations obtained in the LCLV with (a) a sinusoidal and (b) a pulsed signal; $V_{0}=60 \mathrm{Vrms}$.

To characterize the full response of the LCLV we have measured the index change and derived the corresponding LC average orientation angle $\left\langle\theta>\right.$ when varying the applying bias voltage $V_{0}$. The resulting curves are plotted In Fig.4 (a) and (b), respectively. Here the threshold voltage for the LC molecular orientation to occur is $V_{\text {oTh }} \sim 8 \mathrm{Vrms}$ corresponding to the Fréedericksz transition voltage $V_{F} \sim 1.5$ Vrms across the LC layer. The external voltage at which the LC molecules are at half of their total excursion $\left(\theta=45^{\circ}\right)$ is $V_{0} \sim 15 \mathrm{Vrms}$. For $\mathrm{V}_{0}=60 \mathrm{~V}$ rms the saturation of the $\mathrm{LC}$ orientation process is almost reached. Therefore, the changes of the molecular orientation around this working point are small, but allowing a fast response time. The reduced LC excursion provides a small phase 
modulation due to a limited angular excursion of the LC molecules induced by the low grating fringe contrast. However, this provides enough index variations for efficient detection and grating diffraction. The main advantage of working in this regime of transient dynamic is a short response time, hence a frequency bandwidth larger $(\sim \mathrm{kHz})$ with respect to the usual operating conditions of the LCLV.

For a bias voltage at which a large $\Delta \mathrm{n}$ excursion occurs $\left(V_{0}=18 \mathrm{Vrms}\right)$, the usual cutoff is at $\sim 100 \mathrm{~Hz}$. For a large bias voltage ( $\left.V_{0}=60 \mathrm{Vrms}\right)$, a smaller $\Delta \mathrm{n}$ is obtained, but still detectable diffractive efficiency. Note that the spatial resolution is also improved at high bias voltage, this because only the regions close to the cell boundaries contribute to the refractive index change, resulting in a reduced effective thickness of the LC layer.

We now introduce an amplitude modulation of the signal $S$ by using an electro-optic modulator (EOM) followed by a linear polarizer with its axis parallel to the LC director (Fig. 2). The modulated signal intensity has the form $I_{S}(t)=I_{p}(1+$ $\sin 2 \pi f t$ ), with $I_{p}$ the peak value, and the grating amplitude follows at the modulating frequency, $\Delta \mathrm{n}=\Delta \mathrm{n}(\mathrm{f})$. By setting $f$ in the $\mathrm{kHz}$ range we verify that the LCLV responds in a large frequency band, with sub-millisecond response time. A phase grating of amplitude $\Delta \mathrm{n} \sim 10^{-3}$ is observed at $2 \mathrm{kHz}$ signal modulation. As an example we show in Fig. 5 (a) a sinusoidal phase modulation at $2.4 \mathrm{kHz}$, which is clearly detected by measuring on the probe first order diffraction $I_{0}$. As a second example, we show in Fig. 5 (b) a sequence of pulses with $1.2 \mathrm{kHz}$ modulation and $60 \mathrm{~Hz}$ repetition rate. The sub-millisecond temporal resolution can be appreciated as well as a fairly good grating amplitude $\Delta \mathrm{n}$.

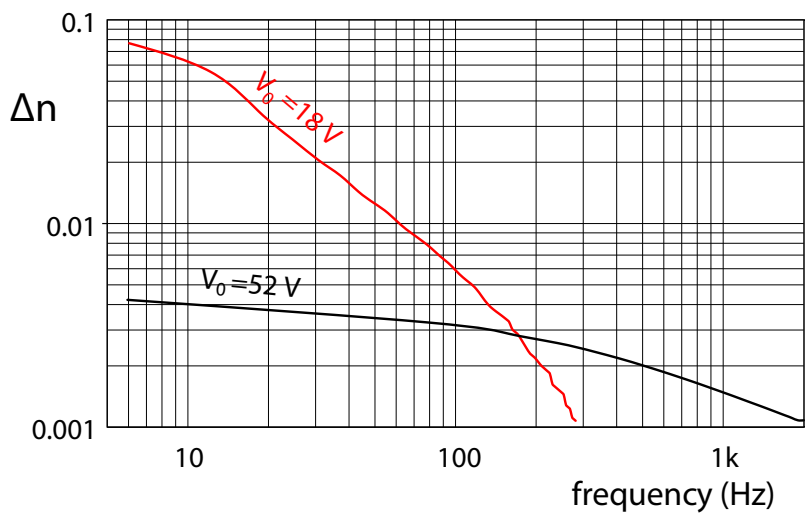

Fig. 6. Frequency response of the LCLV measured for two different working points, $V_{0}=18 \mathrm{Vrms}$ and $V_{0}=52 \mathrm{Vrms}$. The grating amplitude $\Delta \mathrm{n}$ is plotted versus the modulating frequency in log-log scale.

Finally, we have measured the frequency response around the two working points, full span of the LC orientation $\left(V_{0}=18 \mathrm{Vrms}\right)$ and saturation regime $\left(V_{0}=52 \mathrm{Vrms}\right)$. The results are shown in Fig.6. By varying the frequency of a sinusoidal amplitude modulated signal we measure the amplitude $\Delta \mathrm{n}$ of the induced phase grating. It is interesting to note that for the bias voltage at which the full LC orientation is exploited ( $V_{0}=18 \mathrm{Vrms}$ ) the $\Delta \mathrm{n}$ is maximum or $\mathrm{f}<$ $10 \mathrm{~Hz}$ but the frequency response decreases rapidly beyond $\sim 180 \mathrm{~Hz}$, where the crossover with the saturation regime ( $V_{0}=52 \mathrm{Vrms}$ ) occurs. On the contrary, in the saturation regime the dynamics is stretched but the response time decreases only of a factor 10 after the crossover, allowing the detection of phase modulations in the $\mathrm{kHz}$ frequency bandwidth. Note that extension of the method to the near infrared (NIR) can be implemented for LCLV using a NIR sensitive photoconductor, for example a semiinsulating GaAs, which has a high dark resistivity and, therefore, allows applying high bias voltages.

In conclusion, by working in the transient dynamic mode, close to the saturation regime of its response, the LCLV provides submilliseconds phase/amplitude modulations with $\Delta \mathrm{n} \sim 10^{-3}$ grating amplitudes. This provides sufficient dynamics for the detection and processing of the diffracted optical signals. Moreover, the LCLV is a spatially extended optically addressed modulator allowing the detection on large size laser beam and working with low intensities of the addressing beams, $<10 \mathrm{~mW} / \mathrm{cm}^{2}$. These unique properties allow applications in two-wave mixing, dynamic holography and optical phase conjugation with $\mathrm{kHz}$ frequency bandwidth. The conditions of small index modulations where a sub-millisecond response is required may also be well suited for coherent imaging through biomedical tissues or turbid media $[14,15]$.

Funding. Not applicable.

Acknowledgments. Not applicable.

Disclosures. The authors declare no conflicts of interest.

Data availability. Data underlying the results presented in this paper are not publicly available at this time but may be obtained from the authors upon reasonable request.

Supplemental document. Not applicable

\section{References}

1. R.H. Chen, Liquid crystal displays: fundamental physics and technology, (Wiley, Hoboken, New Jersey 2011).

2. I.C. Khoo, Liquid crystals, (Hoboken, New Jersey: John Wiley \& Sons; 2007).

3. U. Efron and G. Liverscu, Spatial light modulator technology: materials, devices and applications, (New York: Dekker; 1995).

4. P. G. de Gennes and AJ. Prost, The physics of liquid crystals, (Oxford: Clarendon Press; 1995).

5. Shin-Tson Wu, Journal of Applied Physics 60, 1836-1838 (1986).

6. Shin-Tson Wu and Chiung-Sheng Wu, J. Appl. Phys. 65, 527-532 (1989).

7. Shin-Tson Wu and Chiung-Sheng Wu, Applied Physics Letters 53, 17941796 (1988).

8. Keyvan Sayyah, Chiung-Sheng Wu, Shin-Tson Wu, and Uzi Efron, Appl. Phys. Lett. 61, 883-885 (1992).

9. U. Bortolozzo, S. Residori, and J.P. Huignard, J. of Holography and Speckles 5, 1-8 (2009).

10. S. Residori, U. Bortolozzo, and J. P. Huignard, Phys. Rev. Lett. 100, 203603-4 (2008).

11. U. Bortolozzo, S. Residori, and J.P. Huignard, Opt. Lett. 35, 2076-2078 (2010).

12. S. Residori, U. Bortolozzo, and J.P. Huignard, Liquid Crystal Reviews 6, 116 (2018).

13. Lili Pei, Dajie Huang, Wei Fan, He Cheng, and Xuechun Li, Appl. Sci. 2018, 8, 1812-11 (2018).

14. Salma Farahi, Emilie Benoit, Alexander A. Grabar, Jean-Pierre Huignard, and Francois Ramaz, Opt Lett 37, 2754-2756 (2012).

15. Yan Liu, Puxiang Lai, Cheng Ma, Xiao Xu, Alexander A. Grabar, and Lihong V. Wang., Nat Commun 6, 5904-9 (2015). 


\section{References}

1. R.H. Chen, Liquid crystal displays: fundamental physics and technology, (Wiley, Hoboken, New Jersey 2011).

2. I.C. Khoo, Liquid crystals, (Hoboken, New Jersey: John Wiley \& Sons; 2007).

3. U. Efron and G. Liverscu, Spatial light modulator technology: materials, devices and applications, (New York: Dekker; 1995).

4. P. G. de Gennes and AJ. Prost, The physics of liquid crystals, (Oxford: Clarendon Press; 1995).

5. Shin-Tson $\mathrm{Wu}$, Phase retardation dependent optical response time of parallel-aligned liquid crystals, Journal of Applied Physics 60, 1836-1838 (1986).

6. Shin-Tson $\mathrm{Wu}$ and Chiung-Sheng $\mathrm{Wu}$, High-speed liquid-crystal modulators using transient nematic effect, J. Appl. Phys. 65, 527-532 (1989).

7. Shin-Tson $\mathrm{Wu}$ and Chiung-Sheng $\mathrm{Wu}$, Small angle relaxation of highly deformed nematic liquid crystals, Applied Physics Letters 53, 1794-1796 (1988).

8. Keyvan Sayyah, Chiung-Sheng Wu, Shin-Tson Wu, and Uzi Efron, Anomalous liquid crystal undershoot effect resulting in a nematic liquid crystal-based spatial light modulator with one millisecond response time, Appl. Phys. Lett. 61, 883-885 (1992).

9. U. Bortolozzo, S. Residori, and J.P. Huignard, Adaptive holography and slow-light in liquid crystal light valves, J. of Holography and Speckles $\mathbf{5}$, 1-8 (2009).

10. S. Residori, U. Bortolozzo, and J. P. Huignard, Slow and Fast Light in Liquid Crystal Light Valves, Phys. Rev. Lett. 100, 203603-4 (2008).

11. U. Bortolozzo, S. Residori, and J.P. Huignard, Slow-light birefringence and polarization interferometry, Opt. Lett. 35, 2076-2078 (2010).

12. S. Residori, U. Bortolozzo, and J.P. Huignard, Liquid crystal light valves as optically addressed liquid crystal spatial light modulators: optical wave mixing and sensing applications, Liquid Crystal Reviews 6, 1-16 (2018).

13. Lili Pei, Dajie Huang, Wei Fan, He Cheng, and Xuechun Li, Phase-Only Optically Addressable Spatial-Light Modulator and On-Line PhaseModulation Detection System, Appl. Sci. 2018, 8, 1812-11 (2018).

14. Salma Farahi, Emilie Benoit, Alexander A. Grabar, Jean-Pierre Huignard, and Francois Ramaz, Time resolved three-dimensional acousto-optic imaging of thick scattering media, Opt Lett 37, 2754-2756 (2012).

15. Yan Liu, Puxiang Lai, Cheng Ma, Xiao Xu, Alexander A. Grabar, and Lihong V. Wang., Optical focusing deep inside dynamic scattering media with near-infrared time-reversed ultrasonically encoded (TRUE) light, Nat Commun 6, 5904-9 (2015). 